\title{
Conductance and density of states as the Kramers-Kronig dispersion relation
}

\author{
Tooru Taniguchi \\ Département de Physique Théorique, Université de Genève, CH-1211, Genève 4, Switzerland
}

(November 3, 2018)

\begin{abstract}
By applying the Kramers-Kronig dispersion relation to the transmission amplitude a direct connection of the conductance with the density of states is given in quantum scattering systems connected to two one-channel leads. Using this method we show that in the Fano resonance the peak position of the density of states is generally different from the position of the corresponding conductance peak, whereas in the Breit-Wigner resonance those peak positions coincide. The lineshapes of the density of states are well described by a Lorentz type in the both resonances. These results are verified by another approach using a specific form of the scattering matrix to describe scattering resonances.
\end{abstract}

Pacs numbers: 72.10.-d, 73.63.Kv, 76.20.+q

\section{INTRODUCTION}

The developments of nano-scale fabrication technique made possible confining electrons in a small region so that the system shows a discrete energy spectrum. Such an electron system is called the quantum dot, whose characteristics have been inzestigated in many theoretical and experimental works 1 . 2

One way by which characteristics of a quantum dot can be investigated is to connect leads to it and to measure its conductance. Many such experiments have actually been carried out and have shown sharp peaks of the conductance or the staircase structure in electric currents as a function of gate voltage or source-drain voltage. 16 These experimental results about conductance peaks have been interpreted on the hypothesis that the electric current through the quantum dot occurs if there is at least one of the energy levels of the quantum dot between chemical potentials of the reservoirs connected to the quantum dot via leads. This hypothesis is justified if the peak position of the conductance coincides with the corresponding peak position of the density of states in the quantum dot.

In this paper we investigate this hypothesis about the peak positions of the conductance and the density of states. We consider a quantum dot connected to two onechannel leads, and assume that the system has a timereversal symmetry. In this system, from the scattering matrix the conductance and the density of states are culated by using the Landauer conductance formulat 11 and the Friedel sum rule 2214 , respectively. Moreover, in order to connect the conductance with the density of states we use the Kramers-Kronig dispersion relation. The Kramers-Kronig dispersion relation connects the real part of a function with its imaginary part by the Hilbert transformation, based on the analyticity of the function. By applying this relation to the logarithm of a scattering matrix element we obtain formulas allowing us to calculate the conductance from the density of states and to calculate the density of states from the conductance.
These formulas are used to investigate a relation of peak positions of the conductance and the density of states.

We consider two kinds of resonances which are called the Breit-Wigner resonance and the Fano resonance. The Breit-Wigner resonance 15 is characterized as the conductance lineshape

$$
G_{b}(E)=\Lambda_{b} \frac{1}{\left(E-E_{0}\right)^{2}+\Delta^{2}}
$$

of a Lorentz type around a resonant energy $E_{0}$ as a function of energy $E$, where $\Lambda_{b}$ is a positive constant. Here the real constant $\Delta$ represents a coupling strength of the quantum dot with leads, and takes a small value compared with energy level spacings of the quantum dot in a weak coupling case with leads. This resonance lineshape agreed with experimental results for conductance in some quantum dots.6 Fig. 11 shows this conductance lineshape with the parameter values $E_{0}=100, \Lambda_{1}=1$ and $\Lambda_{b}=1$. On the other hand, the Fano resonance $\frac{1}{6}$ is characterized by the conductance lineshape

$$
G_{f}(E)=\Lambda_{f} \frac{\left(E-E_{0}+Q\right)^{2}}{\left(E-E_{0}\right)^{2}+\Delta^{2}}
$$

around a resonant energy $E_{0}$, where $\Lambda_{f}$ is a positive constant. Here the parameter $Q$ determines asymmetry in the conductance lineshape of the Fano resonance. The Fano resonance lineshapes are drawn in Fig. 2 with the parameter values $E_{0}=100$ and $\Delta=1$. Here, we chose the parameter $\Lambda_{f}$ as $\Delta^{2} /\left(\Delta^{2}+Q^{2}\right)$ so that the peak value of the conductance is one. The Fano resonance is caused by coupling discrete states with continuous states, and exhibits conductance zerp-points like the energy point $E=E_{0}-Q$ in Eq. (2) 17,18 It should be noted that the Fano resonance is attributed to the Breit-Wigner type in the case of $|Q / \Delta|>>1$ (See Fig. 2 (a).). The conductance lineshape of the Fano resonance is actually observed experimentally by using the scanning tunneling microscopy 2121 and in quantum dot 22 .23. These experimental results show that even the case of $|Q / \Delta|<<1$ like Fig. 2 (c) can happen. 


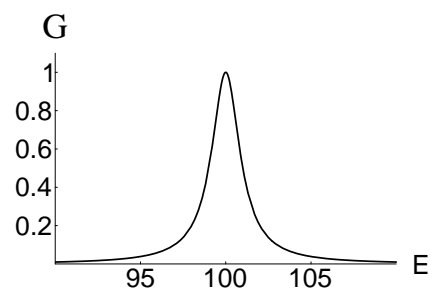

FIG. 1. Conductance lineshape of the Breit-Wigner resonance as a function of energy.

By applying our formula using the Kramers-Kronig dispersion relation to these two kinds of resonances we obtain the following results: (1) In the Breit-Wigner resonance the peak position of the density of states coincides with the position $E=E_{0}$ of the conductance peak. (2) In the Fano resonance the density of states are independent of value of the asymmetric parameter $Q$ in a weak coupling case with leads, and the peak position of the density of states is at $E=E_{0}$. Eq. (2) shows that the peak position of the conductance depends on the asymmetric parameter $Q$ and is given by $E=E_{0}+\Delta^{2} / Q$. Therefore, in the case of $|Q| \Delta \mid>>1$ the peak position of the density of states is close to the position of the conductance peak. On the other hand in the case of $|Q / \Delta|<<1$ the peak position of the density of states is rather close to the energy $E_{0}-Q$ at which the conductance takes a minimum value. We also show that in both resonance types the lineshapes of the density of states are a Lorentz type. These results are correct even in the case where an electron-electron interaction like the charging effect inside the quantum dot plays an important role, because the Friedel sum rule is correct even in presence of electron-electron interactions. 13

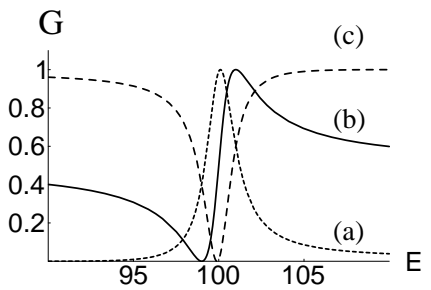

FIG. 2. Conductance lineshapes of the Fano resonance as functions of energy. The graphs (a), (b) and (c) are corresponding to the case of $Q=10,1$ and 0.1 , respectively.

We verify the above results by another approach which does not use the Kramers-Kronig dispersion relation. It is an approach using a specific form of the scattering matrix to describe scattering resonances. We show that only the Breit-Wigner and the Fano resonances are derived from this scattering matrix. By applying the Landauer conductance formula and the Friedel sum rule to this specific form of the scattering matrix we calculate the density of states and the conductance, and obtain the same results as with the dispersion relation approach.

\section{KRAMERS-KRONIG DISPERSION RELATION IN THE TRANSMISSION AMPLITUDE}

The system which we consider in this paper is the quantum dot connected to particle reservoirs via two onedimensional leads. We neglect the effect of a magnetic field so that the system has the time-reversal symmetry. For such a system the scattering matrix $S(E)=\left(S_{l l^{\prime}}(E)\right)$ is represented as a $2 \times 2$ symmetric and unitary matrix at any energy $E$. The conductance $G(E)$ is given by the Landauer conductance formula

$$
G(E)=\frac{q^{2}}{2 \pi \hbar}|t(E)|^{2}
$$

with the charge $q$ of the particle, the Planck constant $2 \pi \hbar$ and the transmission amplitude $t(E) \equiv S_{12}(E)(=$ $\left.S_{21}(E)\right)$. The density of states $\rho(E)$ is given by the Friedel sum rule

$$
\rho(E)=\frac{1}{\pi} \frac{\partial \theta_{f}(E)}{\partial E}
$$

which $\theta_{f}(E)$ is the Friedel phase defined by

$$
\theta_{f}(E) \equiv \frac{1}{2 i} \ln \operatorname{Det}\{S(E)\} .
$$

It is important to note that the Friedel phase $\theta_{f}(E)$ and the transmission amplitude phase $\theta_{t}(E)(\equiv \operatorname{Arg}\{t(E)\})$ are not completely independent. Actually, if the conductance is not zero in any value of energy, then the transmission amplitude phase $\theta_{t}(E)$ is simply given by $\theta_{f}(E)+\pi / 2$. On the other hand, if the conductance takes zero in some energy points $E=E^{(n)}, n=1,2, \cdots$, then the transmission amplitude phase can have discontinuities of $\pm \pi$ in those points, and is connected to the Friedel phase $\theta_{f}(E)$ as $\theta_{t}(E) \overline{-} \theta_{f}(E)+\eta(E)$ with $\eta(E) \equiv \nu+\pi \sum_{n} \gamma_{n} \Theta\left(E-E^{(n)}\right) .24$ Here $\nu$ is an energyindependent constant, $\Theta(x)$ is the step function of $x$, and $\gamma_{n}$ is a constant taking the value $-1,0$ or 1 only. In this paper we treat the conductance like Eq. (2), so we should make up our formula based on the case where there is a conductance zero point.

Since the transmission amplitude phase $\theta_{t}(E)$ has discontinuities in the conductance zero points, we should not assume that the logarithm of the transmission amplitude $t(E)$ itself is an analytic function of energy. Therefore, in order to apply the Kramers-Kronig dispersion relation in which the analyticity of the function plays an essential role, we must carefully remove the singularity caused by the conductance zero points from the logarithm of the transmission amplitude $t(E)$. For this purpose we represent the transmission amplitude $t(E)$ as 


$$
t(E)=\lim _{\varepsilon \rightarrow+0} e^{i \eta(E)} \cdot e^{2^{-1} \ln \left(\varepsilon+|t(E)|^{2}\right)+i \theta_{f}(E)} .
$$

The limit $\varepsilon \rightarrow+0$ is introduced to avoid the divergences of the function $\ln |t(E)|^{2}$ of $E$ in the conductance zero points. In addition, the function $\theta_{f}(E)$ of $E$ is a continuous function because its derivative gives the density of states $\rho(E)$ multiplied by $\pi$, which should be a continuous function of energy. Therefore we get the function $2^{-1} \ln \left(\varepsilon+|t(E)|^{2}\right)+i \theta_{f}(E)$, which can be assumed to be a continuous function of energy.

In the next step we separate its asymptotic form from the transmission amplitude and we make a function which goes to zero as the energy $E$ goes to infinity. For this purpose we introduce the asymptotic forms of the functions $|t(E)|^{2}$ and $\theta_{f}(E)$ as

$$
\begin{gathered}
|t(E)|^{2} \stackrel{E \rightarrow+\infty}{\sim} T^{(\infty)}(E) \\
\theta_{f}(E) \stackrel{E \rightarrow+\infty}{\stackrel{\sim}{\sim}} \theta_{f}^{(\infty)}(E)
\end{gathered}
$$

As an example of the asymptotic transmission amplitude, in the one-dimensional system we may take $t(E) \stackrel{E \rightarrow+\infty}{\sim}$ $\exp (i k l)$, where $l$ is the length of the system and $k$ is the wave vector $\sqrt{2 m E} / \hbar$ with the mass $m$ of the particle, so this gives $T^{(\infty)}(E)=1$ and $\theta_{f}^{(\infty)}(E)=k l$. The transmission amplitude $t(E)$ is represented as

$$
\begin{aligned}
t(E)=\lim _{\varepsilon \rightarrow+0} e^{2^{-1} \ln \left(\varepsilon+T^{(\infty)}(E)\right)} & \\
& \quad \times e^{i\left\{\theta_{f}^{(\infty)}(E)+\eta(E)\right\}} \cdot e^{\Phi_{\varepsilon}(E)}
\end{aligned}
$$

where $\Phi_{\varepsilon}(E)$ is the imaginary function defined by

$$
\begin{aligned}
\Phi_{\varepsilon}(E) \equiv \frac{1}{2} \ln & \frac{\varepsilon+|t(E)|^{2}}{\varepsilon+T^{(\infty)}(E)} \\
& +i\left[\theta_{f}(E)-\theta_{f}^{(\infty)}(E)\right] .
\end{aligned}
$$

An important characteristic of the function $\Phi_{\varepsilon}(E)$ is that this function satisfies the condition

$$
\lim _{E \rightarrow+\infty} \Phi_{\varepsilon}(E)=0
$$

and can be assumed to be a continuous function of energy. The real part of the function $\Phi_{\varepsilon}(E)$ gives the conductance

$$
G(E)=\lim _{\varepsilon \rightarrow+0} G^{(\infty)}(E) e^{2 \operatorname{Re}\left\{\Phi_{\varepsilon}(E)\right\}}
$$

by using Eq. (3), where $G^{(\infty)}(E)$ is the conductance $\left(q^{2} /(2 \pi \hbar)\right) T^{(\infty)}(E)$ in the high energy limit. Using Eq. (仼) the density of states $\rho(E)$ is connected to the imaginary part of the function $\Phi_{\varepsilon}(E)$ by

$$
\rho(E)=\rho^{(\infty)}(E)+\lim _{\varepsilon \rightarrow+0} \frac{1}{\pi} \frac{\partial \operatorname{Im}\left\{\Phi_{\varepsilon}(E)\right\}}{\partial E}
$$

where $\rho^{(\infty)}(E)$ is the asymptotic form of the density of states in the high energy limit and is given by $(1 / \pi) \partial \theta_{f}^{(\infty)}(E) / \partial E$. Now we finish preparing the function $\Phi_{\varepsilon}(E)$ to which we apply the Kramers-Kronig dispersion relation.

So far, the function $\Phi_{\varepsilon}(E)$ has been defined only in the real energy region $(0,+\infty)$. (Here we took the origin of energy so that the lower bound of the energy is zero.) Now, in order to apply the Kramers-Kronig dispersion relation to the function $\Phi_{\varepsilon}(E)$, we extend this function so that it is defined in the whole upper half plain of the imaginary number $E$ including the real axis. We assume that such an extension can be done under the three conditions:

(I) The function $\Phi_{\varepsilon}(E)$ of $E$ is analytic in the whole upper half plain and in the real axis in the imaginary number $E$.

(II) $\lim _{|E| \rightarrow+\infty}\left|\Phi_{\varepsilon}(E)\right|=0$ in any energy $E$ satisfying $\operatorname{Im}\{E\} \geq 0$.

(III) $\Phi_{\varepsilon}(-E)=\Phi_{\varepsilon}(E)^{*}$ in any real number $E$.

It should be noted that the condition (II) is a generalization of Eq. (11). In this paper we choose the value $\theta_{f}(0)-\theta_{f}^{(\infty)}(0)$ as 0 , so that the right-hand side and the left hand side in the equation of the condition (III) coincide at the origin $E=0$. Known as the Kramers-Kronig dispersion relation 25 using the conditions (I), (II) and (III) the real part and the imaginary part of the function $\Phi_{\varepsilon}(E)$ are connected as

$$
\begin{gathered}
\operatorname{Re}\left\{\Phi_{\varepsilon}(E)\right\}=\frac{2}{\pi} \hat{\mathcal{P}} \int_{0}^{+\infty} d E^{\prime} \frac{E^{\prime} \operatorname{Im}\left\{\Phi_{\varepsilon}\left(E^{\prime}\right)\right\}}{E^{\prime 2}-E^{2}} \\
\operatorname{Im}\left\{\Phi_{\varepsilon}(E)\right\}=-\frac{2}{\pi} \hat{\mathcal{P}} \int_{0}^{+\infty} d E^{\prime} \frac{E \operatorname{Re}\left\{\Phi_{\varepsilon}\left(E^{\prime}\right)\right\}}{E^{\prime 2}-E^{2}}
\end{gathered}
$$

where the operator $\hat{\mathcal{P}}$ means to take the principal integral in the following integral.

Using Eqs. (10), (12) and (13), the relations (14) and (15) lead to a direct connection between the conductance and the density of states:

$$
\begin{array}{r}
G(E)=G^{(\infty)}(E) \exp \left\{-\int_{0}^{+\infty} d E^{\prime} \mathcal{C}\left(E, E^{\prime}\right)\right. \\
\left.\times\left[\rho\left(E^{\prime}\right)-\rho^{(\infty)}\left(E^{\prime}\right)\right]\right\} \\
\rho(E)=\rho^{(\infty)}(E)+\lim _{\varepsilon \rightarrow+0} \int_{0}^{+\infty} d E^{\prime} \mathcal{D}\left(E, E^{\prime}\right) \\
\times \ln \frac{\varepsilon+G\left(E^{\prime}\right)}{\varepsilon+G^{(\infty)}\left(E^{\prime}\right)}
\end{array}
$$

where the functions $\mathcal{C}(x, y)$ and $\mathcal{D}(x, y)$ of $x$ and $y$ are defined by 


$$
\begin{aligned}
& \mathcal{C}(x, y) \\
& \equiv \lim _{\epsilon \rightarrow+0} \ln \left\{\left[(x-y)^{2}+\epsilon^{2}\right]\left[(x+y)^{2}+\epsilon^{2}\right]\right\} \\
& \mathcal{D}(x, y) \equiv-\lim _{\epsilon \rightarrow 0} \frac{1}{2 \pi^{2}}\left\{\frac{(x-y)^{2}-\epsilon^{2}}{\left[(x-y)^{2}+\epsilon^{2}\right]^{2}}\right. \\
& \left.+\frac{(x+y)^{2}-\epsilon^{2}}{\left[(x+y)^{2}+\epsilon^{2}\right]^{2}}\right\} .
\end{aligned}
$$

(See Appendix A about the derivations of these equations.) Here, in order to derive Eq. (16) we assumed $\lim _{E \rightarrow+\infty}\left\{\theta_{f}(E)-\theta_{f}^{(\infty)}(E)\right\} \ln E=0$, which is stronger than the condition (8). Eqs. (16) and (17) are the key results of this paper.

As a general feature of the conductance shown by using Eq. (16) the conductance $G(E)$ is invariant under the change $\rho(E) \rightarrow \rho(E)+\alpha$ (So $\rho^{(\infty)}(E) \rightarrow \rho^{(\infty)}(E)+\alpha$.) of the density of states in any constant $\alpha$. Similarly Eq. (17) implies that the density of states $\rho(E)$ is invariant under the change $G(E) \rightarrow \beta G(E)$ in any constant $\beta$.

\section{APPLICATION TO THE BREIT-WIGNER AND FANO RESONANCE}

In this section, using Eq. (17) we calculate the densities of states in the Breit-Wigner resonance and the Fano resonance. In the actual calculation we use the equation

$$
\begin{aligned}
& \rho(E)-\rho^{(\infty)}(E) \\
&=-\lim _{\varepsilon \rightarrow+0} \frac{1}{\pi^{2}} \hat{\mathcal{P}} \int_{0}^{+\infty} d E^{\prime} \frac{E^{\prime}}{E^{\prime 2}-E^{2}} \\
& \quad \times \frac{\partial}{\partial E^{\prime}} \ln \frac{G_{\varepsilon}\left(E^{\prime}\right)}{G_{\varepsilon}^{(\infty)}\left(E^{\prime}\right)}
\end{aligned}
$$

with $G_{\varepsilon}(E) \equiv \varepsilon+G(E)$ and $G_{\varepsilon}^{(\infty)}(E) \equiv \varepsilon+G^{(\infty)}(E)$. Eq. (20) is equivalent with Eq. (17), as shown in the end of Appendix A.

Before calculating the density of states, we consider some problems in applications of the formula (20) to the conductances (11) and (2). First, strictly speaking, in order to obtain the density of states using the formula (20) we need to know the value of the conductance in any energy $E$. On the other hand Eqs. (11) and (2) are correct only around the resonant energy $E_{0}$. However the integral kernel $E^{\prime} /\left(E^{\prime 2}-E^{2}\right)$ in the formula (20) has a large absolute value only around $E^{\prime}=E$, so the value of conductance around the energy $E_{0}$ is enough to obtain approximately the density of states around the energy $E_{0}$.

The second problem in applications of the formula (20) is that we do not know the general asymptotic forms of the conductance and the density of states, which is needed to calculate the exact form of the density of states $\rho(E)$ by using Eq. (20). In this section we assume that the energy dependence of the asymptotic form of the transmission amplitude is the same as the onedimensional case, namely $t(E) \stackrel{E \rightarrow+\infty}{\sim} \exp (i \lambda \sqrt{E})$ using a constant $\lambda$. Therefore the asymptotic form of the conductance and the density of states are given by $G^{(\infty)}(E)=$ $q^{2} /(2 \pi \hbar)$ and $\rho^{(\infty)}(E)=\lambda /(2 \pi \sqrt{E})$, respectively. We do not have to care whether the conductances (11) and (2) satisfy the condition $\lim _{E \rightarrow \infty} G(E)=q^{2} /(2 \pi \hbar)$, because these forms of the conductances are justified only around the resonant energy $E_{0}$.

It is valuable to extract an essential part giving a peak of the density of states from the right-hand side of Eq. (20). For this purpose we rewrite Eq. (20) as

$$
\begin{gathered}
\rho(E)=-\lim _{\varepsilon \rightarrow+0} \frac{1}{2 \pi^{2}} \hat{\mathcal{P}} \int_{-\infty}^{+\infty} d E^{\prime} \frac{1}{E^{\prime}-E} \frac{\partial \ln G_{\varepsilon}\left(E^{\prime}\right)}{\partial E^{\prime}} \\
+\mathcal{F}(E)+\frac{\lambda}{2 \pi \sqrt{E}}
\end{gathered}
$$

Here we used the specified asymptotic form of the conductance and the density of states, and $\mathcal{F}(E)$ is defined by

$$
\mathcal{F}(E) \equiv-\lim _{\varepsilon \rightarrow+0} \frac{1}{2 \pi^{2}} \int_{0}^{+\infty} d E^{\prime} \frac{\Xi_{\varepsilon}\left(E^{\prime}\right)}{E^{\prime}+E}
$$

with $\Xi_{\varepsilon}(E) \equiv(\partial / \partial E) \ln \left[G_{\varepsilon}(E) / G_{\varepsilon}(-E)\right]$. The function $\mathcal{F}(E)$ of $E$ is estimated as

$$
|\mathcal{F}(E)|<\lim _{\varepsilon \rightarrow+0} \frac{1}{2 \pi^{2}} \frac{1}{E} \int_{0}^{+\infty} d E^{\prime}\left|\Xi_{\varepsilon}\left(E^{\prime}\right)\right| .
$$

We consider a weak coupling case of the quantum dot with leads, so we may regard the constant $\Delta$ as a small parameter compared with energy level spacings of the quantum dot. In this case we can assume that the energy value $E_{0}$ is large enough compared with the constant $|\Delta|$. Noting that it is enough for us to calculate the density of states $\rho(E)$ only around the energy $E_{0}$, we estimate that the contribution of the function $\mathcal{F}(E)$ to the density of states is negligible around the energy $E_{0}$ under the condition that the integral $\int_{0}^{+\infty} d E\left|\Xi_{\varepsilon}(E)\right|$ has a finite value, because of the small factor $1 / E \simeq 1 / E_{0}$ in the right-hand side of Eq. (23). The third term in the right-hand side of Eq. (21) is a monotonous decreasing function of energy, so this part is also negligible in a large energy value $E \simeq E_{0}$ and almost does not contribute to changes of the peak position and the configuration of the density of states. Therefore the main contribution to the peak of the density of states comes only from the first term in the right-hand side of Eq. (21).

\section{A. Breit-Wigner resonance}

Fig. 3 is the Breit-Wigner resonance lineshape (11) and the corresponding density of states which is calculated by using Eq. (20). Here we chose the parameters as 
$\lambda=1$, and the other parameter values are the same as in Fig. 1. Fig. 3 shows that the peak position of the density of states coincides with the peak position of the conductance in the Breit-Wigner resonance.

Now we check this result by the analytical consideration based on Eq. (21) neglecting its second and third terms. Substituting Eq. (1) into Eq. (21) we obtain the density of states as

$$
\begin{aligned}
\rho(E) & \simeq \frac{1}{\pi^{2}} \hat{\mathcal{P}} \int_{-\infty}^{+\infty} d E^{\prime} \frac{1}{E^{\prime}-E} \frac{E^{\prime}-E_{0}}{\left(E^{\prime}-E_{0}\right)^{2}+\Delta^{2}} \\
& =\frac{1}{\pi} \frac{|\Delta|}{\left(E-E_{0}\right)^{2}+\Delta^{2}} .
\end{aligned}
$$

This implies that the density of states is a Lorentz type whose peak position is at $E=E_{0}$ and is independent of the value of the prefactor $\Lambda_{b}$ in the conductance lineshape (1).

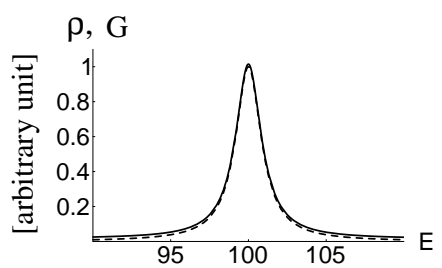

FIG. 3. Density of states (solid line) and the conductance (dashed line) as functions of energy in the Breit-Wigner resonance.

\section{B. Fano resonance}

The conductance (2) in the Fano resonance is an example in which a conductance zero occurs, so the infinitesimal constant $\varepsilon$ in Eq. (20) plays an important role in calculating the density of states.

Fig. A is the density of states corresponding to the Fano resonane lineshape (2), which is calculated by using Eq. (20).26 Here, we chose the parameters as $\lambda=1$ and the other other parameter values are the same as in Fig. 2. In the case of $|Q / \Delta|>>1$ (See Fig. 4 (a).), where the conductance lineshape is close to the BreitWigner type, the peak position of the density of states is close to the peak position of the conductance. On the other hand, in the case of $|Q / \Delta|<<1$ (See Fig. A (c).), where the conductance lineshape is a gulf rather than a peak, the peak position of the density of states is rather close to the gulf of the conductance.

Now we calculate the density of states $\rho(E)$ by using Eq. (21) neglecting its second and third terms. After a small calculation (See Appendix B for the detail of the calculation) we obtain $\rho(E) \simeq(1 / \pi)|\Delta| /\left\{\left(E-E_{0}\right)^{2}+\right.$ $\left.\Delta^{2}\right\}$, which is a Lorentz type with the peak at the energy $E_{0}$ and is the same as with the case of the Breit-Wigner resonance. It should be emphasized that this form of the density of states is independent of the value of the asymmetric parameter $Q$ and the prefactor $\Lambda_{f}$ in the conductance lineshape (2).


FIG. 4. Density of states (solid line) and the conductance (dashed line) as functions of energy in the Fano resonance. The graphs (a), (b) and (c) are corresponding to the cases of $Q=10,1$ and 0.1 , respectively. The arrow in each graph shows the position of the conductance peak.

\section{ANOTHER APPROACH USING A SPECIFIC FORM OF SCATTERING MATRIX}

In this section, using another approach which does not use the Kramers-Kronig dispersion relation, we verify our results obtained in the previous section.

The specific form of the scattering matrix

$$
S(E)=A\left(I+i B \frac{1}{E-E_{0}+i \Delta}\right)
$$

around a resonant energy $E_{\text {pas }}$ been proposed to describe scattering resonances.27 28 Here, the $2 \times 2$ matrix $A \equiv\left(A_{l l^{\prime}}\right)$ is introduced as an energy-independent scattering matrix in the high energy limit, so that it is an unitary matrix in itself: $A A^{\dagger}=A^{\dagger} A=I$. The matrix $B \equiv\left(B_{l l^{\prime}}\right)$ is also an energy-independent $2 \times 2$ matrix and satisfies the conditions

$$
B^{\dagger}=B \text { and } B(B+2 I \Delta)=0,
$$

so that the scattering matrix $S(E)$ given by Eq. 25) becomes an unitary matrix in any energy $E$. It may be 
noted that either $B=-2 I \Delta$ or $B=0$ satisfy the condition (26), but this gives an energy-independent conductance which is not pertinent to the subject of this paper. Therefore in this section we assume $B \neq-2 I \Delta$ and $B \neq 0$, which lead to the parameterized representation

$$
B=\Delta \cdot\left(\begin{array}{cc}
-1+\sin \phi & e^{i \varphi} \cos \phi \\
e^{-i \varphi} \cos \phi & -1-\sin \phi
\end{array}\right)
$$

of the matrix $B$ with real parameters $\phi$ and $\varphi$.

By applying the Friedel sum rule (14) to the scattering matrix (25) the density of states $\rho(E)$ is given by

$$
\rho(E)=\frac{1}{\pi} \frac{\Delta}{\left(E-E_{0}\right)^{2}+\Delta^{2}},
$$

which satisfies $\lim _{\Delta \rightarrow+0} \rho(E)=\delta\left(E-E_{0}\right) .29$ Therefore the resonance lineshape of the density of states is always a Lorentz type with a peak at the energy $E_{0}$. This agrees with the result concerning the lineshape of the density of states in the previous section.

Now we check that the scattering matrix (25) gives the conductance lineshapes of the Breit-Wigner and the Fano resonance, and consider a relation between the peak positions of the conductance and the density of states. First we consider the case of $A_{12}=0$. In this case using Eqs. (3) and (25) the conductance $G(E)$ is represented as the Breit-Wigner type (11) with $\Lambda_{b}=q^{2}\left|B_{12}\right|^{2} /(2 \pi \hbar)$ by noting $\left|A_{11}\right|=1$, and the density of states is connected to the conductance simply as $\rho(E)=\Delta \cdot G_{b}(E) /\left(\pi \Lambda_{b}\right)$. In this case the peak position of the conductance is at $E=E_{0}$ and coincides with the peak position of the density of states.

Second we consider the case of $A_{12} \neq 0$. In this case, by applying the Landauer conductance formula (3) to the scattering matrix (25) we obtain the conductance

$$
G(E)=W_{f}+\Lambda_{f} \frac{\left(E-E_{0}+Q\right)^{2}}{\left(E-E_{0}\right)^{2}+\Delta^{2}}
$$

of the Fano type with parameter values

$$
\begin{gathered}
W_{f}=\frac{q^{2}}{2 \pi \hbar}\left|A_{12}\right|^{2} \mathcal{K}, \\
\Lambda_{f}=\frac{q^{2}}{2 \pi \hbar}\left|A_{12}\right|^{2}(1-\mathcal{K}), \\
Q=-\frac{d_{1}}{1-\mathcal{K}} .
\end{gathered}
$$

Here $\mathcal{K}$ is defined by

$$
\mathcal{K} \equiv \frac{\Delta^{2}+d_{1}^{2}+d_{2}^{2}-\sqrt{\left(\Delta^{2}+d_{1}^{2}-d_{2}^{2}\right)^{2}+4 d_{1}^{2} d_{2}^{2}}}{2 \Delta^{2}}
$$

and $d_{j}, j=1,2$ are introduced as

$$
\begin{gathered}
d_{1} \equiv \operatorname{Im}\left\{\frac{A_{11}}{A_{12}} B_{12}\right\}, \\
d_{2} \equiv \Delta+B_{22}+\operatorname{Re}\left\{\frac{A_{11}}{A_{12}} B_{12}\right\} .
\end{gathered}
$$

It is important to note that the constant $\mathcal{K}$ satisfies the inequality $0 \leq \mathcal{K} \leq 1$ so the constants $W_{f}$ and $\Lambda_{f}$ given by Eqs. (30) and (31) are not negative. The peak position of the conductance in this case is at $E=E_{0}+\Delta^{2} / Q$, which does not coincide with the peak position $E=E_{0}$ of the density of states shown by Eq. (28). The Fano conductance (29) takes a minimum value at the energy $E_{0}-Q$. Therefore the peak position $E=E_{0}$ of the density of states is close to the peak position of the conductance in the case of $|Q / \Delta|>>1$, but as the quantity $|Q / \Delta|$ goes to 0 it moves closer to the energy at which the conductance takes a minimum value. This is exactly the same result as in the previous section.

The above results in this section are independent of the time-reversal symmetry of the system and are correct even in presence of a magnetic field. However if the system has the time reversal symmetry and the conditions $S_{12}=S_{21}$ and $A_{12}=A_{21}$ are satisfied, then we obtain $W_{f}=0, \Lambda_{f}=\left(q^{2} /(2 \pi \hbar)\right)\left|A_{12}\right|^{2}$ and $Q=-d_{1}$ (See Appendix C for their proofs.). Therefore the conductance (29) becomes exactly the same form as Eq. (2) in the time-reversal symmetric system.

\section{CONCLUSION AND REMARKS}

In this paper using the Kramers-Kronig dispersion relation, the Landauer conductance formula and the Friedel sum rule we have discussed a method to calculate the density of states from conductance and to calculate conductance from the density of states in quantum scattering systems connected to two one-channel leads. We considered the case of no magnetic field, so that the system had the time-reversal symmetry. Our formula was applied to the Breit-Wigner resonance and the Fano resonance, and led to their profiles of the density of states. In the BreitWigner resonance the peak positions of the conductance and the density of states coincide. On the other hand, in the Fano resonance, a relation of the peak positions of the conductance and the density of states depends on the parameter $|Q / \Delta|$ which determines asymmetry of the conductance lineshape. In the case of $|Q / \Delta|>>1$ the peak position of the density of states is close to the position of the conductance peak, like the Breit-Wigner resonance. However in the case of $|Q / \Delta|<<1$ the peak position of the density of states is rather close to the energy at which the conductance takes a minimum value. We also showed that the lineshape of the density of states is a Lorentz type in both resonances. These results 
are model-independent, and are correct even if electronelectron interaction inside the quantum dot plays an important role. These results were verified by another consideration which does not use the Kramers-Kronig dispersion relation but uses a specified form of the scattering matrix to describe scattering resonances.

The relation between peak positions of the conductance and the density of states is important to explain an in-phase characteristic of the transmission amplitude phase (See Eq. (13) in Ref. 24), which has been measured actually in an experiment. 0 Some works indicated that the Fano resonance property is important to cause this phenomenon. 31.32 In Ref. 24 it has already been shown that in a simple model consisting of a branch connected to a one-dimensional perfect wire the peak positions of the density of states are in gulfs of the conductance.

The advantage of our approach using the KramersKronig dispersion relation is that we can know the density of states directly from the conductance which can be measured in experiments. We can also calculate the density of states from the scattering matrix itself, but it is extremely difficult for the scattering matrix itself to be measured in experiments. On the other hand, one of the disadvantages of the dispersion relation approach is that this approach is justified only under some restrictive conditions, for example, two one-channel leads, no magnetic field, the conditions (I), (II) and (III), etc. We would get a wrong result if we neglected these conditions. For example, the approach in the section 4 predicts a nonzero constant $W_{f}$ in presence of a magnetic field, and if we were to apply the formula (17) to such a nonzero $W_{f}$ case then the density of states would take a negative value in an energy region, which is not correct. To reduce the number of these conditions in our dispersion relation approach is one of the important future problems.

\section{ACKNOWLEDGMENTS}

I wish to thank M. Büttiker for providing a stimulating environment for the present work. I acknowledge a careful reading of this paper by M. Honderich.

\section{APPENDIX A: DERIVATION OF THE CONNECTION BETWEEN THE CONDUCTANCE AND THE DENSITY OF STATES}

In this appendix we give the derivation of Eqs. 16), (17) and (20).

First we should notice the equation

$$
\begin{aligned}
\hat{\mathcal{P}} & \frac{1}{E^{\prime 2}-E^{2}} \\
& =\frac{1}{2 E^{\prime}}\left(\hat{\mathcal{P}} \frac{1}{E^{\prime}-E}+\hat{\mathcal{P}} \frac{1}{E^{\prime}+E}\right)
\end{aligned}
$$

$$
\begin{aligned}
& =\lim _{\epsilon \rightarrow 0} \frac{1}{2 E^{\prime}}\left[\frac{E^{\prime}-E}{\left(E^{\prime}-E\right)^{2}+\epsilon^{2}}\right. \\
& \left.+\frac{E^{\prime}+E}{\left(E^{\prime}+E\right)^{2}+\epsilon^{2}}\right] \text {. }
\end{aligned}
$$

Similarly we obtain

$$
\begin{aligned}
& \hat{\mathcal{P}} \frac{1}{E^{\prime 2}-E^{2}} \\
&=\lim _{\epsilon \rightarrow 0} \frac{1}{2 E}\left[\frac{E^{\prime}-E}{\left(E^{\prime}-E\right)^{2}+\epsilon^{2}}\right. \\
&\left.\quad-\frac{E^{\prime}+E}{\left(E^{\prime}+E\right)^{2}+\epsilon^{2}}\right] .
\end{aligned}
$$

It follows from Eqs. (10), (12), (14), 18) and (A.1) that

$$
\begin{aligned}
& \ln \frac{G(E)}{G^{(\infty)}(E)}=\lim _{\varepsilon \rightarrow+0} 2 \operatorname{Re}\left\{\Phi_{\varepsilon}(E)\right\} \\
& =\lim _{\varepsilon \rightarrow+0} \frac{4}{\pi} \hat{\mathcal{P}} \int_{0}^{+\infty} d E^{\prime} \frac{E^{\prime} \operatorname{Im}\left\{\Phi_{\varepsilon}\left(E^{\prime}\right)\right\}}{E^{\prime 2}-E^{2}} \\
& =\lim _{\varepsilon \rightarrow+0} \lim _{\epsilon \rightarrow 0} \frac{2}{\pi} \int_{0}^{+\infty} d E^{\prime}\left[\frac{E^{\prime}-E}{\left(E^{\prime}-E\right)^{2}+\epsilon^{2}}\right. \\
& \left.\quad+\frac{E^{\prime}+E}{\left(E^{\prime}+E\right)^{2}+\epsilon^{2}}\right] \operatorname{Im}\left\{\Phi_{\varepsilon}\left(E^{\prime}\right)\right\} \\
& =-\int_{0}^{+\infty} d E^{\prime} \frac{\partial \mathcal{C}\left(E, E^{\prime}\right)}{\partial E^{\prime}}\left[\theta_{f}\left(E^{\prime}\right)-\theta_{f}^{(\infty)}\left(E^{\prime}\right)\right] \\
& =-\int_{0}^{+\infty} d E^{\prime} \mathcal{C}\left(E, E^{\prime}\right) \frac{1}{\pi} \frac{\partial\left[\theta_{f}\left(E^{\prime}\right)-\theta_{f}^{(\infty)}\left(E^{\prime}\right)\right]}{\partial E^{\prime}} \\
& =E^{\prime}\left(E, E^{\prime}\right)\left[\rho(E)-\rho^{(\infty)}(E)\right]
\end{aligned}
$$

where we used the conditions $\theta_{f}(0)-\theta_{f}^{(\infty)}(0)=0$ and $\lim _{E^{\prime} \rightarrow+\infty} \mathcal{C}\left(E, E^{\prime}\right)\left[\theta_{f}\left(E^{\prime}\right)-\theta_{f}^{(\infty)}\left(E^{\prime}\right)\right]=0$. This leads to Eq. (16). Similarly, using Eqs. (10), (13), (15), (19) and (A.2) we obtain

$$
\begin{aligned}
& \rho(E)-\rho^{(\infty)}(E)=\lim _{\varepsilon \rightarrow+0} \frac{1}{\pi} \frac{\partial \operatorname{Im}\left\{\Phi_{\varepsilon}(E)\right\}}{\partial E} \\
& =-\lim _{\varepsilon \rightarrow+0} \frac{2}{\pi^{2}} \frac{\partial}{\partial E} \hat{\mathcal{P}} \int_{0}^{+\infty} d E^{\prime} \frac{E \operatorname{Re}\left\{\Phi_{\varepsilon}\left(E^{\prime}\right)\right\}}{E^{\prime 2}-E^{2}} \\
& =-\lim _{\varepsilon \rightarrow+0} \lim _{\epsilon \rightarrow 0} \frac{1}{\pi^{2}} \frac{\partial}{\partial E} \int_{0}^{+\infty} d E^{\prime}\left[\frac{E^{\prime}-E}{\left(E^{\prime}-E\right)^{2}+\epsilon^{2}}\right. \\
& \left.\quad-\frac{E^{\prime}+E}{\left(E^{\prime}+E\right)^{2}+\epsilon^{2}}\right] \operatorname{Re}\left\{\Phi_{\varepsilon}\left(E^{\prime}\right)\right\} \\
& =\lim _{\varepsilon \rightarrow+0} \int_{0}^{+\infty} d E^{\prime} \mathcal{D}\left(E, E^{\prime}\right) \ln \frac{\varepsilon+G\left(E^{\prime}\right)}{\varepsilon+G^{(\infty)}\left(E^{\prime}\right)}
\end{aligned}
$$

This leads to Eq. (17).

Using the expression (A.4) we obtain another expression for a relation between the density of states and the conductance: 


$$
\begin{gathered}
\rho(E)-\rho^{(\infty)}(E) \\
=\lim _{\varepsilon \rightarrow+0} \lim _{\epsilon \rightarrow 0} \frac{1}{\pi^{2}} \int_{0}^{+\infty} d E^{\prime} \\
\quad \times\left\{\frac { \partial } { \partial E ^ { \prime } } \left[\frac{E^{\prime}-E}{\left(E^{\prime}-E\right)^{2}+\epsilon^{2}}\right.\right. \\
\left.\left.\quad+\frac{E^{\prime}+E}{\left(E^{\prime}+E\right)^{2}+\epsilon^{2}}\right]\right\} \operatorname{Re}\left\{\Phi_{\varepsilon}\left(E^{\prime}\right)\right\} \\
=\lim _{\varepsilon \rightarrow+0} \int_{0}^{+\infty} d E^{\prime} \tilde{\mathcal{D}}\left(E, E^{\prime}\right) \\
\times \frac{\partial}{\partial E^{\prime}} \ln \frac{\varepsilon+G\left(E^{\prime}\right)}{\varepsilon+G(\infty)\left(E^{\prime}\right)}
\end{gathered}
$$

where $\tilde{\mathcal{D}}\left(E, E^{\prime}\right)$ is defined by

$$
\begin{aligned}
\tilde{\mathcal{D}}\left(E, E^{\prime}\right) \equiv-\lim _{\epsilon \rightarrow 0} \frac{1}{2 \pi^{2}}[ & \frac{E^{\prime}-E}{\left(E^{\prime}-E\right)^{2}+\epsilon^{2}} \\
& \left.+\frac{E^{\prime}+E}{\left(E^{\prime}+E\right)^{2}+\epsilon^{2}}\right]
\end{aligned}
$$

and satisfies the condition $\tilde{\mathcal{D}}(E, 0)=0$. Eqs. A.1 and (A.6) lead to Eq. (20).

\section{APPENDIX B: DENSITY OF STATES IN THE FANO RESONANCE}

In this appendix we calculate the density of states by using Eqs. (2) and (21). Neglecting its second and third term, Eq. (21) leads to the density of states $\rho(E)$ :

$$
\rho(E) \simeq-\lim _{\varepsilon \rightarrow+0} \frac{1}{2 \pi^{2}} \hat{\mathcal{P}} \int_{-\infty}^{+\infty} d E^{\prime} \frac{\Gamma_{\varepsilon}\left(E^{\prime}\right)}{E^{\prime}+E_{0}-E}
$$

where the function $\Gamma_{\varepsilon}(E)$ of $E$ is introduced as

$$
\begin{aligned}
\Gamma_{\varepsilon}(E) \equiv & \frac{1}{G_{\varepsilon \Lambda_{f}}\left(E+E_{0}\right)} \frac{\partial G_{\varepsilon \Lambda_{f}}\left(E+E_{0}\right)}{\partial E} \\
= & -2 \frac{(E+Q)\left(Q E-\Delta^{2}\right)}{\left(E^{2}+\Delta^{2}\right)\left[(E+Q)^{2}+\varepsilon\left(E^{2}+\Delta^{2}\right)\right]} \\
= & -2 \frac{E}{E^{2}+\Delta^{2}} \\
& +2 \frac{E+\frac{Q}{1+\varepsilon}}{\left(E+\frac{Q}{1+\varepsilon}\right)^{2}+\varepsilon\left[\frac{\Delta^{2}}{1+\varepsilon}+\left(\frac{Q}{1+\varepsilon}\right)^{2}\right]} .
\end{aligned}
$$

Using the formula

$$
\frac{1}{\pi} \hat{\mathcal{P}} \int_{-\infty}^{+\infty} d y \frac{1}{y-x} \frac{y}{y^{2}+a^{2}}=\frac{|a|}{x^{2}+a^{2}}
$$

for a real constant $a$, it follows from Eqs. B.1) and (B.2) that

$$
\rho(E) \simeq \frac{1}{\pi} \frac{|\Delta|}{\left(E-E_{0}\right)^{2}+\Delta^{2}}
$$

in $E \neq E_{0}-Q$.

\section{APPENDIX C: TIME REVERSAL SYMMETRY IN THE FANO RESONANCE}

In this appendix we show $d_{2}=0$ under the conditions $A_{12}=A_{21} \neq 0$ and $S_{12}=S_{21}$. This result $d_{2}=0$ leads to $\mathcal{K}=0$, so we obtain $W_{f}=0, \Lambda_{f}=\left(q^{2} /(2 \pi \hbar)\right)\left|A_{12}\right|^{2}$ and $Q=-d_{1}$ by using Eqs. (30), (31) and (32).

The matrix $A$, which is an unitary matrix, is represented as

$$
A=\left(\begin{array}{cc}
i e^{i\left(\tilde{\theta}+\tilde{\varphi}_{1}\right)} \sin \tilde{\phi} & e^{i\left(\tilde{\theta}+\tilde{\varphi}_{2}\right)} \cos \tilde{\phi} \\
e^{i\left(\tilde{\theta}-\tilde{\varphi}_{2}\right)} \cos \tilde{\phi} & i e^{i\left(\tilde{\theta}-\tilde{\varphi}_{1}\right)} \sin \tilde{\phi}
\end{array}\right)
$$

with real parameters $\tilde{\theta}, \tilde{\varphi}_{j}, j=1,2$ and $\tilde{\phi}$. The condition $A_{12}=A_{21}$ imposes

$$
\tilde{\varphi}_{2}=0 \text { or } \pi
$$

The condition $S_{12}=S_{21}$ under Eq. C.2 implies that the multiplied matrix $A B$ is also symmetric. This leads to the condition

$$
\tan \phi=-\frac{\sin \left(\varphi+\tilde{\varphi}_{1}\right)}{\cos \tilde{\varphi}_{2}} \tan \tilde{\phi}
$$

On the other hand the constant $d_{2}$ given by Eq. (35) is represented as

$$
\begin{aligned}
d_{2}=-\Delta \cdot & \cos \phi \cdot[\tan \phi \\
& \left.+\sin \left(\varphi+\tilde{\varphi}_{1}-\tilde{\varphi}_{2}\right) \cdot \tan \tilde{\phi}\right] .
\end{aligned}
$$

By using Eqs. (C.2), C.3) and (C.4) we obtain $d_{2}=0$.

(1) M. A. Kastner, Phys. Today, 46, 24 (1993).

(2) L. P. Kouwenhoven, C. M. Marcus, P. L. Mceuen, S. Tarucha, R. M. Westervelt, and N. S. Wingreen, L. L. Sohn et al. (eds.), Mesoscopic Electron Transport, Kluwer Academic Publishers, p105-214, (1997).

(3) M. A. Reed, J. N. Randall, R. J. Aggarwal, R. J. Matyi, T. M. Moore, and A. E. Wetsel, Phys. Rev. Lett. 60, 535 (1988).

(4) J. H. F. Scott-Thomas, S. B. Field, M. A. Kastner, H. I. Smith, and D. A. Antoniadis, Phys. Rev. Lett. 62, 583 (1989).

(5) L. P. Kouwenhoven, N. C. van der Vaart, A. T. Johnson, W. Kool, C. J. P. M. Harmans, J. G. Williamson, A. A. M. Staring, and C. T. Foxon, Z. Phys. B, 85, 367 (1991). 
(6) E. B. Foxman, P. L. McEuen, U. Meirav, N. S. Wingreen, Y. Meir, P. A. Belk, N. R. Belk, M. A. Kastner, and S. J. Wind, Phys. Rev. B 47, 10020 (1993).

(7) R. Landauer, Philos. Mag. 21, 863 (1970).

(8) E. N. Economou and C. M. Soukoulis, Phys. Rev. Lett. 46, 618 (1981).

(9) D. S. Fisher and P. A. Lee, Phys. Rev. B23, 6851 (1981).

(10) M. Büttiker, Y. Imry, R. Landauer, and S. Pinhas, Phys. Rev. B31, 6207 (1985).

(11) M. Büttiker, Phys. Rev. Lett. 57, 1761 (1986).

(12) J. Friedel, Philos. Mag. 43, 153 (1952).

(13) J. S. Langer and V. Ambegaokar, Phys. Rev. 121, 1090 (1961).

(14) R. Dashen, S. -k. Ma, and H. J. Bernstein, Phys. Rev. 187, 345 (1969).

(15) G. Breit and E. Wigner, Phys. Rev. 49, 519 (1936).

(16) U. Fano, Phys. Rev. 124, 1866 (1961).

(17) E. Tekman and P. F. Bagwell, Phys. Rev. B 48, 2553 (1993).

(18) J. U. Nöckel and A. D. Stone, Phys. Rev. B 50, 17415 (1994).

(19) J. Li, W. Schneider, R. Berndt, and B. Delley, Phys. Rev. Lett. 80, 2893 (1998).

(20) V. Madhavan, W. Chen, T. Jamneala, M. F. Crommie, and N. S. Wingreen, Science 280, 567, (1998).

(21) H. C. Manoharan, C. P. Lutz, and D. M. Eigler, Nature, 403, 512 (2000).

(22) J. Göres, D. Goldhaber-Gordon, S. Heemeyer, M. A. Kastner, H. Shtrikman, D. Mahalu, and U. Meirav, Phys.
Rev. B 62, 2188 (2000).

(23) I. G. Zacharia, D. Goldhaber-Gordon, G. Granger, M. A. Kastner, Yu. B. Khavin, H. Shtrikman, D. Mahalu, and U. Meirav, cond-mat/0009140 (unpublished).

(24) T. Taniguchi and M. Büttiker, Phys. Rev. B 60, 13814 (1999).

(25) See, for example, C. J. Joachain, Quantum Collision Theory (North-Holland Publishing Company 1975).

(26) Strictly speaking from a mathematical point of view, the right-hand side of Eq. (20) gives a delta functional singularity in the conductance zero point $E=E_{0}-Q$, but this singularity is not a real contribution to the density of states. (Here it should be emphasized again that the density of states is a continuous function of energy in the open conductor.) Therefore we neglect this singularity in the results of calculation, and define the value of the density of states at the energy $E_{0}-Q$ as $\rho\left(E_{0}-Q\right) \equiv \lim _{E \rightarrow E_{0}-Q} \rho(E)$.

(27) J. R. Taylor, Scattering Theory (Wiley, New York 1972).

(28) H. -W. Lee, Phys. Rev. Lett. 82, 2358 (1999).

(29) Eq. (28) implies that the constant $\Delta$ must be positive because the density of states given by Eq. (28) must be positive. Therefore the scattering matrix as a function of $E$ is analytic in the whole upper half plain and in the real axis in the imaginary number $E$.

(30) R. Schuster, E. Buks, M. Heiblum, D. Mahalu, V. Umansky, and H. Shtrikman, Nature, 385, 417 (1997).

(31) H. Xu and W. Sheng, Phys. Rev. B 57, 11903 (1998).

(32) C. -M. Ryu and S. Y. Cho, Phys. Rev. B 58, 3572 (1998). 